\title{
Diversidade de membracídeos (Hemiptera, Membracidae) e sobreposição de recursos tróficos em área do semi-árido
}

\author{
Antonio J. Creão-Duarte ${ }^{1}$, Ulisses U. Anjos ${ }^{2} \&$ Wellington E. Santos ${ }^{1}$ \\ 1. Departamento de Sistemática e Ecologia, Universidade Federal da Paraíba, Campus I, 58.051-900, João Pessoa, PB, Brasil. creaoduarte@dse.ufpb.br; well-bio@hotmail.com \\ 2. Departamento de Estatística, Universidade Federal da Paraíba, Campus I, 58.051-900, João Pessoa, PB, Brasil. ulisses@de.ufpb.br
}

\begin{abstract}
Diversity and trophic niche overlap of membracids (Hemiptera, Membracidae) in a semi-arid area. We examined 1,069 individuals of 13 species of membracids collected on their host plants, between August 2004 and September 2005, in a semi-arid region, State of Paraíba, Northeastern Brazil. Enchenopa concolor (Fairmaire, 1846) (31.6\%) and E. euniceae Creão-Duarte \& Rothéa, 2006 (24.8\%) were the most abundant species. Five families of host plants harbored these insects. Amongst these families, Fabaceae showed the highest richness (12) and abundance (70.3\%) of membracids. Darnis olivacea Fabricius, 1803, Hygris beckeri Sakakibara, 1998 and Sundarion flavum (Fairmaire, 1846) showed the highest values of niche breadth. Amongst the most abundant species, E. minuta Creão-Duarte \& Rothéa, 2006 and Micrutalis binaria (Faimaire, 1846) showed the highest trophic niche overlap, but the temporal overlap between them was relatively low. The results revealed possible strategies developed by the species for coexistence and utilization of resources in Caatinga.
\end{abstract}

KEYWORDS. Caatinga, host plants, niche overlap.

RESUMO. Foram examinados 1.069 indivíduos de 13 espécies de membracídeos coletados em suas plantas hospedeiras, entre agosto de 2004 e setembro de 2005, em uma região do semi-árido da Paraíba, Nordeste do Brasil. Enchenopa concolor (Fairmaire, 1846) (31,6\%) e E. euniceae Creão-Duarte \& Rothéa, $2006(24,8 \%)$ foram as espécies mais abundantes. Cinco famílias de plantas hospedeiras abrigaram esses insetos. Entre essas famílias, Fabaceae apresentou maior riqueza (12) e abundância (70,3\%) de membracídeos. Darnis olivacea Fabricius, 1803, Hygris beckeri Sakakibara, 1998 e Sundarion flavum (Fairmaire, 1846) apresentaram os maiores valores de amplitude de nicho. Entre as espécies mais abundantes, E. minuta Creão-Duarte \& Rothéa, 2006 e Micrutalis binaria (Faimaire, 1846) mostraram a maior sobreposição de nicho trófico, mas a sobreposição temporal entre elas foi relativamente baixa. Os resultados revelaram possíveis estratégias desenvolvidas pelas espécies para coexistência e exploração de recursos na Caatinga.

PALAVRAS-CHAVE. Caatinga, plantas hospedeiras, sobreposição de nicho.

A Caatinga é o único bioma restrito quase que totalmente ao nordeste do território brasileiro, onde se estende por nove estados, ocupando uma área em torno de $850.000 \mathrm{~km}^{2}$, umas das maiores áreas de Floresta Neotropical Estacional Seca da América do Sul (DiRzo et al., 2011). A grande extensão, os tipos de clima e solo e a multiplicidade nas formas de relevo do semiárido, traduz-se em diferentes paisagens do domínio das caatingas, como os vales úmidos, as chapadas sedimentares e as amplas superfícies pediplanadas que podem explicar a razão da flora possuir elevado grau de variabilidade (SANTANA \& Souto, 2006).

Esse bioma encontra-se altamente fragmentado e degradado pelas atividades agropecuárias extensivas e o avanço das cidades. $\mathrm{O}$ desconhecimento de sua diversidade biológica e, particularmente, da entomofauna tem motivado estudos de inventário, taxonomia e ecologia que buscam, de um modo geral, disponibilizar dados que possam ser úteis em estratégias de conservação e de exploração sustentável dos recursos naturais. Embora esses estudos sejam ainda raros, informações sobre alguns grupos taxonômicos de Insecta estão disponíveis (VIANA, 1999; IANNUZZi et al., 2003; LeAl et al., 2003; MaIA et al., 2003; Gusmão \& CReÃo-Duarte, 2004; Hernández, 2007; VASCONCELlos et al., 2010a,b; SANTOs et al., 2011; Aguiar et al., 2012; SANTOS et al., 2012).

Em nenhum ambiente as espécies são igualmente comuns, em geral o que se verifica é a alta abundância de poucas espécies e uma grande parcela de espécies raras (MACARThUR, 1965). A avaliação de distribuição de abundância de espécies facilita o entendimento sobre os processos que determinam a diversidade biológica, pois, em certa medida, a abundância reflete o sucesso da espécie em competir por recursos limitados (MAGURRAN, 2011) e medidas de diversidade são fundamentais para a compreensão e o monitoramento das alterações verificadas nos ecossistemas, sejam elas causadas por fenômenos naturais ou antrópicos (LAwTON et al., 1998).

Em estudos de ecologia de comunidades é útil quantificar o quanto duas espécies se sobrepõem na utilização de espaço, alimento e outros recursos (Hurlbert, 1978; ThOMPson, 2012), já que pode haver organismos mais especializados que outros, sendo possível avaliar quantitativamente medidas de tamanho e de ocupação de nicho. Do mesmo modo, medir a sobreposição dos recursos utilizados conjuntamente pelas espécies de uma guilda, por exemplo, é um dos primeiros passos para entender como a comunidade está organizada (KREBS, 1999; BLÜTHGEN \& KLEIN, 2011).

Membracidae é uma família com cerca de 3.200 espécies descritas. Apresenta uma classificação relativamente estável e bem documentada, em decorrência da predominância de trabalhos de taxonomia que imperam neste grupo. Esses insetos estabelecem interações com plantas, de onde sugam seiva, e com outros insetos, sobretudo formigas, que assistem determinadas espécies que formam colônias (FunKHOUSER, 1950; Wood, 1993; Lin et al., 2004). 
Estudos dessas interações vêm recebendo maior atenção no Brasil em anos recentes (DEL-CLARO \& Oliveira, 1993, 1999, 2000; Del-Claro, 2004; Fernandes et al., 2005; Lopes, 1995; Moreira \& DelClaro, 2005; Del-Claro et al., 2006), todavia, apesar deste esforço, é inquestionável a necessidade de se reunir informações sobre esse táxon e o seu papel nos ecossistemas tropicais, visto sua extrema diversidade taxonômica, morfológica, ecológica e comportamental (Wood, 1993), especialmente em ecossistemas semi-áridos como a Caatinga, que possui em sua dinâmica pulsos de estresse hídrico, com reflexos na disponibilidade de recursos para os organismos fitófagos (VAsconcellos et al., 2010a). Dessa forma, este estudo objetivou analisar a diversidade de Membracidae e suas plantas hospedeiras, como também avaliar a amplitude e sobreposição de nicho trófico e temporal desses insetos em uma área de Caatinga.

\section{MATERIAL E MÉTODOS}

O estudo de campo foi realizado na Reserva Particular do Patrimônio Natural (RPPN) Fazenda Almas, uma área de 3.500 ha localizada no município de São José dos Cordeiros (7²8'45’'s; 3654'18'W), Paraíba, Brasil, distante cerca de $300 \mathrm{~km}$ da capital, João Pessoa. Esta área localiza-se na região do Cariri Paraibano, que se encontra em uma depressão de 200 a 300 metros do nível do planalto da Borborema, caracterizada por uma formação de Caatinga arbórea e arbustiva (ANDRADE-LIMA, 1981).

O clima da região caracteriza-se por apresentar condições extremas dentre os padrões meteorológicos, como elevada radiação, baixa umidade relativa e baixa nebulosidade (PRADO, 2003). A precipitação anual é inferior a $600 \mathrm{~mm}$ e irregular, sendo o clima classificado como 'semi-árido quente' (Bsh), com chuvas de verão, segundo classificação de Köppen. As temperaturas médias anuais são elevadas, entre 26 e $30^{\circ} \mathrm{C}$. A estação chuvosa normalmente concentra-se em três meses no primeiro semestre (PRADO, 2003) e o período seco dura de seis a nove meses, podendo estender-se até dez meses (PARAÍBA, 1985).

Foram realizadas seis amostragens, três no ano de 2004 (agosto, outubro e dezembro) e três em 2005 (março, maio e setembro). Cada amostragem foi resultado de 10 horas de vistorias, sendo cada hora o resultado da soma do tempo de esforço de captura investido por um ou dois coletores, ou seja, 60 ou 30 minutos/coletor, respectivamente. As amostragens foram realizadas ao longo de trilhas nas bordas da vegetação nativa mais elevada, vistoriando-se todas as plantas hospedeiras, sendo a mesma área amostrada em todas as coletas.

As capturas foram conduzidas manualmente utilizando-se frascos coletores ou sacos plásticos, estes últimos empregados na coleta das espécies que formam colônias para evitar fuga de espécimes. Para as espécies já bem representadas nas coletas adotou-se o procedimento apenas de registro.

A identificação dos insetos foi realizada mediante comparação com fotos de material-tipo, bibliografia especializada ou por comparação com exemplares da Coleção Entomológica do Departamento de Sistemática e Ecologia da Universidade Federal da Paraíba. Após identificação, os espécimes foram incorporados ao acervo da referida coleção. A identificação das plantas foi executada por comparação com material depositado no herbário Professor Lauro Pires Xavier, da mesma instituição.

A distribuição das espécies em classe de constância é utilizada em análise faunística para informar a ocorrência das espécies ao longo do período de coleta. As espécies presentes em mais de $50 \%$ das coletas foram designadas constantes $(\mathrm{Ct})$, as presentes entre 25 e $50 \%$, acessórias (Ac), e aquelas presentes em menos de 25\%, acidentais (Ad), de acordo com Bodenheimer (SILVEIRANeto et al., 1976). Foram definidas três classes de abundância com base no intervalo de confiança para média, ao nível de 90\%. São abundantes (A) as espécies cujo total de indivíduos é maior que o limite superior do intervalo de confiança; comuns $(\mathrm{C})$ o total de indivíduos situa-se entre os limites superior e inferior do intervalo e raras $(\mathrm{R})$ o total de indivíduos é menor que o limite inferior do intervalo de confiança. A abundância relativa de cada espécie na comunidade é o percentual com que cada uma participa com relação ao total de indivíduos coletados.

Os valores de amplitude de nicho foram calculados pelo índice de Levin, como em MAJUMdar et al. (2011); o referido índice tem valor máximo quando igual número de indivíduos de uma espécie está associado com cada um dos estados de recurso. Isso implica que esta espécie não discrimina entre os estados de recurso (ou discrimina, mas as prefere igualmente) e por essa razão têm a maior amplitude de nicho (Cowell \& Futuyma, 1971). A espécie especialista tem amplitude de nicho igual a zero, enquanto que as espécies que apresentam os maiores valores são as mais generalistas.

A sobreposição de nicho foi calculada usando o índice de Pianka, mediante o emprego do programa Ecological Methodology, versão presente em KREBS (1999). Este índice é simétrico e varia de 0 a 1,0. Quando 0 (zero), indica que o recurso foi utilizado por uma única espécie e 1,0 (um) indica total sobreposição do recurso. A definição do valor a partir do qual se considera que existe sobreposição efetiva de nicho é arbitrária e definida diferentemente por diversos autores (AGUIAR, 2003; Majumdar et al., 2011; CAPello et al., 2012). Neste estudo, valores acima de 0,60 foram interpretados como indicadores de sobreposição. As medidas de sobreposição de nicho foram calculadas apenas para as espécies das classes abundantes e comuns.

As medidas de amplitude e sobreposição de nicho foram baseadas na distribuição de organismos individuais 
(espécimes de membracídeos) associados a um conjunto de estados de recursos (plantas hospedeiras), sendo que a tabela denominada Matriz de Recursos tem as linhas compostas pelas espécies de Membracidae e as colunas, estados de recursos, pelas plantas hospedeiras, como proposto por Colwell \& Futuyma (1971).

\section{RESULTADOS E DISCUSSÃO}

Treze espécies de 11 gêneros de Membracidae e um total de 1.069 indivíduos foram coletados ao longo do estudo. Enchenopa concolor (Fairmaire, 1846) e E. euniceae Creão-Duarte \& Rothéa, 2006 foram as espécies mais abundantes, com $338(31,6 \%)$ e 265 $(24,8 \%)$ indivíduos, respectivamente (Tab. I). Estas espécies e Enchenopa minuta Creão-Duarte \& Rothéa, 2006 foram as únicas que formaram colônias atendidas por formigas. Não há trabalhos de diversidade de Membracidae para o Brasil em que os principais parâmetros de diversidade (riqueza e abundância) sejam analisados em seu conjunto. LOPES (1995) ao avaliar o uso de plantas hospedeiras pelos membracídeos em uma região de Cerrado, registrou 52 espécies desses insetos, porém, não informou sobre a abundância das mesmas. Embora a fauna de Membracidae para a Região Neártica seja mais bem estudada, são poucas as informações sobre a abundância das espécies (MAsON \& Loye, 1981; Johnson \& Freytag, 1997; BartLett et al., 2008).

Enchenopa eunicea ocorreu em todas as seis coletas, enquanto E. concolor foi registrada em cinco e E. minuta em quatro, portanto, todas foram constantes. Sundarion flavum (Fairmaire, 1846), embora classificada como acessória, foi mais abundante que Ceresa vitulus (Fabricius, 1775) e Darnis olivacea Fabricius, 1803, que foram constantes (Tab. I). Observações de espécies acessórias mais abundantes que espécies constantes também foram feitas para Membracidae e Cicadellidae
(Miranda et al., 2009) e para outros grupos de insetos (Ringenberg et al., 2010; Teston \& Delfina, 2010).

Esperava-se para a área estudada que o número de espécies acidentais fosse muito mais elevado que o de espécies constantes em função da influência climática na flutuação populacional (VASCONCELLOS et al., 2010a). Entretanto, $61,5 \%$ das espécies foram constantes, enquanto que $23,1 \%$ foram acessórias e apenas 15,4\% foram acidentais. Esses resultados divergem dos obtidos para outros grupos de insetos, onde as espécies constantes estão sempre em menor número que as acidentais (MARCHIORI et al., 2003; TESTON \& CORSEUIL, 2004; Graciani et al., 2005; D’Avila \& Marchini, 2008; Miranda et al., 2009; Teston \& Delfina, 2010). A localização da área de coleta, às proximidades de um açude, pode ter influenciado os resultados, tendo em vista que as plantas desta área se mantiveram verdes, mesmo na época seca.

Cinco famílias botânicas hospedaram membracídeos na localidade estudada, sendo Fabaceae a principal com $752(70,3 \%)$ indivíduos coletados, seguida por Sapindaceae com 108 (10,1\%), Leguminosae com $92(8,6 \%)$, Malvaceae com $89(8,3 \%)$ e Celastraceae com $28(2,6 \%)$ (Tab. II). À exceção de Hypsoprora coronata (Fabricius, 1803), coletada somente sobre Serjania glabrata Kunth (Sapindaceae), todas as demais espécies ocorreram em Fabaceae. Sapindaceae e Leguminosae abrigaram cinco espécies e Malvaceae e Celastraceae, duas e uma, respectivamente. A associação de membracídeos com suas plantas hospedeiras tem sido avaliada buscando identificar as principais famílias botânicas que abrigam esses insetos. Em vegetação de Cerrado, Lopes (1995) listou Araliaceae, Asteraceae, Leguminosae, Malpighiaceae, Myrtaceae e Nyctaginaceae.

Mimosa tenuiflora (Wild.) Poir. foi a planta que abrigou a maior riqueza de membracídeos, oito, seguida por Bowdichia virgilioides Kunth e M. ophthalmocentra

Tab. I. Distribuição temporal, constância e abundância de membracídeos coletados na RPPN Fazenda Almas, São José dos Cordeiros, Paraíba em 2004 e 2005 (Classes de constância: Ct, constante; Ac, acessória; Ad, acidental. Classes de abundância: A, abundante; C, comum; R, rara).

\begin{tabular}{|c|c|c|c|c|c|c|c|c|c|c|c|c|}
\hline \multirow{2}{*}{$\begin{array}{l}\text { Espécies de } \\
\text { membracídeos }\end{array}$} & \multicolumn{5}{|c|}{ Período Seco } & \multicolumn{3}{|c|}{ Período Chuvoso } & \multicolumn{2}{|c|}{ Total } & \multirow{2}{*}{ Constância } & \multirow{2}{*}{ Abundância } \\
\hline & Ago/2004 & Out/2004 & Dez/2004 & Set $/ 2005$ & Total & Mar/2005 & Mai/2005 & Total & $\mathrm{N}$ & $\%$ & & \\
\hline Enchenopa concolor & & 3 & 61 & 37 & 101 & 180 & 57 & 237 & 338 & 31,62 & $\mathrm{Ct}$ & A \\
\hline Enchenopa euniceae & 2 & 58 & 11 & 37 & 108 & 103 & 54 & 157 & 265 & 24,79 & $\mathrm{Ct}$ & A \\
\hline Enchenopa minuta & & & 44 & 12 & 56 & 133 & 6 & 139 & 195 & 18,24 & $\mathrm{Ct}$ & A \\
\hline Melusinella nervosa & 55 & & & 1 & 56 & 28 & 4 & 32 & 88 & 8,23 & $\mathrm{Ct}$ & $\mathrm{C}$ \\
\hline Thrasymedes pallescens & 4 & 1 & 1 & 34 & 40 & 24 & 14 & 38 & 78 & 7,30 & $\mathrm{Ct}$ & $\mathrm{C}$ \\
\hline Micrutalis binaria & & & 17 & 8 & 25 & 13 & 20 & 33 & 58 & 5,43 & $\mathrm{Ct}$ & $\mathrm{C}$ \\
\hline Sundarion flavum & & & & & 0 & 5 & 9 & 14 & 14 & 1,31 & Ac & $\mathrm{R}$ \\
\hline Ceresa vitulus & 4 & 3 & & 1 & 8 & 1 & 1 & 2 & 10 & 0,94 & $\mathrm{Ct}$ & $\mathrm{R}$ \\
\hline Darnis olivacea & 2 & 1 & 2 & & 5 & 2 & & 2 & 7 & 0,65 & $\mathrm{Ct}$ & $\mathrm{R}$ \\
\hline Hypsoprora coronata & & & 6 & & 6 & & & 0 & 6 & 0,56 & Ad & $\mathrm{R}$ \\
\hline Erosne costrica & & 3 & & & 3 & 1 & & 1 & 4 & 0,37 & $\mathrm{Ac}$ & $\mathrm{R}$ \\
\hline Hygris beckeri & & & & & 0 & 2 & 2 & 4 & 4 & 0,37 & Ac & $\mathrm{R}$ \\
\hline Tolania armata & & & & & 0 & & 2 & 2 & 2 & 0,19 & $\mathrm{Ad}$ & $\mathrm{R}$ \\
\hline Total & 67 & 69 & 142 & 130 & 408 & 492 & 169 & $\overline{661}$ & 1069 & 100 & & \\
\hline
\end{tabular}


Mart. ex Benth., com seis (Fig. 1). Indigofera suffruticosa Mill. e Phaseolus sp. foram as plantas hospedeiras onde se coletou o maior número de indivíduos, $261(24,4 \%)$ e $204(19,1 \%)$, respectivamente, muito embora, cada uma dessas plantas abrigasse apenas três espécies. Enchenopa concolor, a espécie mais abundante, foi comum a essas duas plantas e teve respectivamente $233(68,9 \%)$ e 44 $(13,0 \%)$ indivíduos coletados sobre elas (Tab. II), o que demonstra a importância relativa de I. suffruticosa e Phaseolus sp. para a manutenção deste membracídeo no semi-árido.

Mimosa tenuiflora e $M$. ophthalmocentra foram as únicas plantas onde se coletou D. olivacea e as mais importantes para a permanência de E. euniceae, pois hospedaram $80,4 \%$ dos indivíduos coletados. Caesalpinia pyramidalis Tul. foi a planta mais importante para manutenção de Thrasymedes pallescens (Stål, 1896), pois abrigou 98,7\% dos indivíduos coletados. Sida galheirensis Ulbr., embora abrigando apenas Melusinella nervosa (Fairmaire, 1846), foi muito importante para a permanência dessa espécie, uma vez que $70,5 \%$ de seus indivíduos foram coletados sobre ela. São raros os trabalhos que registram plantas abrigando a diversidade de membracídeos. LOPES (1995) registrou 40 espécies de plantas hospedeiras em vegetação de Cerrado, sendo Byrsonima intermedia A. Juss. (Malpighiaceae) a que abrigou a maior riqueza, 10 espécies. No mesmo bioma, Fernandes et al. (2005) relataram a presença comum de membracídeos sobre B. crassifolia (L.) Kunth. Na Pensilvânia, EUA, Wallace \& Maloney (2010) coletaram 39 espécies e 6.861 indivíduos sobre Quercus ilicifolia Wangenh. (Fagaceae).

A amplitude de nicho de uma espécie pode ser estimada medindo-se a uniformidade da distribuição dos indivíduos daquela espécie entre os estados de recurso (tipos de alimentos, de habitat, substratos etc.), ou seja, o tamanho do nicho de uma espécie pode ser medido observando-se a distribuição dos seus indivíduos no conjunto dos estados do recurso (COLWELl \& Futuyma,

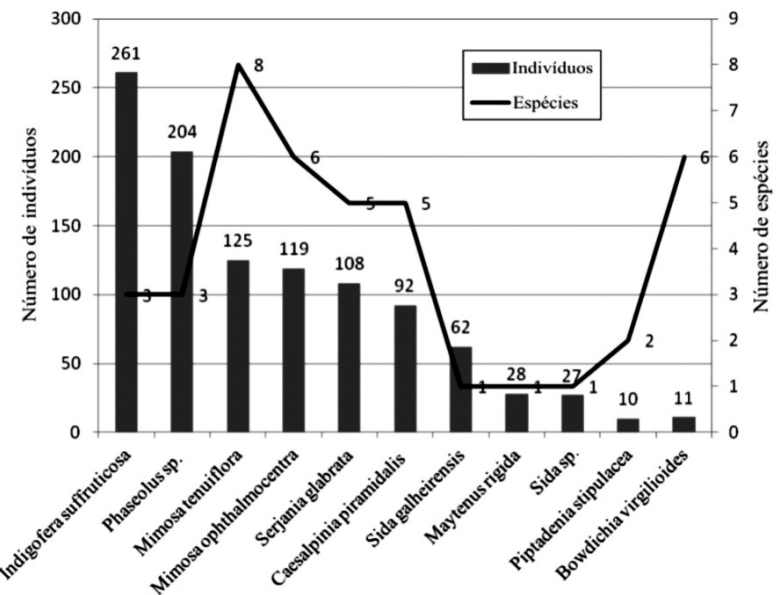

Fig. 1. Número de indivíduos e de espécies de membracídeos coletados sobre plantas hospedeiras na RPPN Fazenda Almas, São José dos Cordeiros, Paraíba em 2004 e 2005.
1971). Darnis olivacea, Hygris beckeri Sakakibara, 1998 e S. flavum foram as espécies com maior amplitude de nicho, isto é, as que melhor se distribuem dentre os recursos por elas selecionados (Tab. II). Enchenopa concolor, apesar de ocorrer em oito plantas hospedeiras, teve tamanho padronizado de nicho reduzido, o qual superou apenas os de $H$. coronata e Tolania armata Goding, 1927, cujos valores foram nulos. Isto ocorre porque E. concolor explora os recursos utilizados de modo desproporcional quando comparada com as demais espécies. Semelhantemente, D'AlmeIDA \& Almeida (1998) observaram que Peckia chrysostoma (Wiedemann, 1830) e Musca domestica (Linnaeus, 1758), apesar de presentes em cinco dos seis estados de recurso disponíveis, apresentaram tamanho de nicho menor que Chrysomya megacephala (Fabricius, 1794), ocorrente em apenas três destes.

Os maiores valores de sobreposição de nicho trófico foram observados para E. minuta com Micrutalis binaria (Faimaire, 1846) $(0,638)$ e para M. nervosa com E. concolor $(0,376)$ (Tab. III). Isto decorre porque essas duplas de espécies exploraram conjuntamente os mesmos recursos alimentares (Tab. II). A exploração dos mesmos recursos em proporções semelhantes e a baixa variabilidade na dieta, entre espécies diferentes, podem levar à sobreposição de nicho, como observado para outros insetos (Tavares \& Williams, 1990; Majumdar et al., 2011; CAPEllo et al., 2012). Vale ressaltar que a sobreposição de nicho não sugere necessariamente competição entre os pares de espécies envolvidas, pois o recurso pode existir em quantidade suficiente para atender as espécies envolvidas (SCHOEREDER \& Coutinho, 1991; D’Almeida \& Almeida, 1998).

Além disso, pares de espécies com alta sobreposição ao longo de uma dimensão de nicho, potencialmente competidoras, frequentemente tem separação de nicho ao longo de uma ou mais das três dimensões: alimento, espaço e/ou tempo (Pianka, 1974; MaY, 1975; CarvalHo \& GOMEs, 2001). Diferenças ecológicas em umas dessas três dimensões de nicho podem reduzir a competição interespecífica e, assim, facilitar a coexistência de diversas espécies (Hanski, 1983; Albrecht \& Gotelli, 2001; Aguiar et al., 2012). Tal fato foi observado nas espécies mais abundantes. Entre essas, a maioria dos pares tiveram valores elevados de sobreposição de nicho temporal, mas esses pares de espécies não sobrepuseram nicho trófico (Tab. III). Enquanto que E. minuta e M. binaria, que apresentaram o maior valor de sobreposição de nicho trófico, apresentaram baixa sobreposição temporal $(0,630)$, considerando a maioria dos valores encontrados. Efeitos da sobreposição de nicho temporal também foram observados em outros grupos de insetos (Prieto \& DahNers, 2009; SAntos \& Presley, 2010; SAntos et al., 2012).

Estes resultados podem ser interpretados como estratégias desenvolvidas pelas espécies de Membracidae para coexistência e exploração de recursos limitados em um ambiente inóspito e fortemente sazonal como o estudado. 
Tab. II. Matriz de recurso em valores absolutos de indivíduos de Membracidae capturados, por planta hospedeira, em coletas realizadas na RPPN Fazenda Almas, São José dos Cordeiros, Paraíba em 2004 e 2005 (Fabaceae: A, Indigofera suffruticosa Mill.; B, Phaseolus sp.; C, Mimosa tenuiflora (Wild.) Poir.; D, Mimosa ophthalmocentra Mart. ex Benth.; E, Senna spectabilis (DC.) H.S. Irwin \& Barneby; F, Bowdichia virgilioides Kunth; G, Piptadenia stipulacea (Benth.) Ducke; Sapindaceae: H, Serjania glabrata Kunth; Leguminosae: I, Caesalpinia pyramidalis Tul.; Malvaceae: J, Sida galheirensis Ulbr.; K, Sida sp.; Celastraceae: L, Maytenus rigida Mart.).

\begin{tabular}{|c|c|c|c|c|c|c|c|c|c|c|c|c|c|c|}
\hline \multirow{2}{*}{ Espécies de membracídeos } & \multicolumn{12}{|c|}{ Plantas hospedeiras } & \multirow{2}{*}{ Total } & \multirow{2}{*}{ Levin $\left(B_{A}\right)$} \\
\hline & A & B & $\mathrm{C}$ & $\mathrm{D}$ & $\mathrm{E}$ & $\mathrm{F}$ & $\mathrm{G}$ & $\mathrm{H}$ & I & $\mathrm{J}$ & $\mathrm{K}$ & $\mathrm{L}$ & & \\
\hline Enchenopa concolor & 233 & 44 & 1 & 3 & 19 & 2 & & 9 & & & 27 & & 338 & 0,141 \\
\hline Enchenopa euniceae & 2 & & 110 & 103 & 2 & 4 & 9 & 2 & 5 & & & 28 & 265 & 0,247 \\
\hline Enchenopa minuta & & 144 & 1 & & & & 1 & 49 & & & & & 195 & 0,214 \\
\hline Melusinella nervosa & 26 & & & & & & & & & 62 & & & 88 & 0,713 \\
\hline Thrasymedes pallescens & & & 1 & & & & & & 77 & & & & 78 & 0,026 \\
\hline Micrutalis binaria & & 16 & & & & & & 42 & & & & & 58 & 0,665 \\
\hline Sundarion flavum & & & 4 & 5 & & 1 & & & 4 & & & & 14 & 0,793 \\
\hline Ceresa vitulus & & & 3 & & 1 & 1 & & & 5 & & & & 10 & 0,593 \\
\hline Darnis olivacea & & & 3 & 4 & & & & & & & & & 7 & 0,960 \\
\hline Hypsoprora coronata & & & & & & & & 6 & & & & & 6 & 0,000 \\
\hline Erosne costrica & & & & 3 & & & & & 1 & & & & 4 & 0,600 \\
\hline Hygris beckeri & & & 2 & 1 & & 1 & & & & & & & 4 & 0,833 \\
\hline Tolania armata & & & & & & 2 & & & & & & & 2 & 0,000 \\
\hline Indivíduos/planta & 261 & 204 & 125 & 119 & 22 & 11 & 10 & 108 & 92 & 62 & 27 & 28 & 1069 & \\
\hline Espécies/planta & 3 & 3 & 8 & 6 & 3 & 6 & 2 & 5 & 5 & 1 & 1 & 1 & & \\
\hline
\end{tabular}

Tab. III. Medidas de sobreposição de nicho trófico (superior direito) e temporal (inferior esquerdo) dos membracídeos mais abundantes coletados na RPPN Fazenda Almas, São José dos Cordeiros, Paraíba em 2004 e 2005, utilizando índice de Pianka.

\begin{tabular}{lcccccc}
\hline Espécies de membracídeos & E. con. & E. eun. & E. min. & M. ner. & T. pal. & M. bin. \\
\hline Enchenopa concolor & $\mathrm{x}$ & 0,026 & 0,186 & 0,376 & 0,000 & 0,100 \\
Enchenopa eunicea & 0,871 & $\mathrm{x}$ & 0,009 & 0,005 & 0,042 & 0,012 \\
Enchenopa minuta & 0,965 & 0,784 & $\mathrm{x}$ & 0,000 & 0,000 & 0,638 \\
Melusinella nervosa & 0,425 & 0,387 & 0,432 & $\mathrm{x}$ & 0,000 & 0,000 \\
Thrasymedes pallescens & 0,723 & 0,763 & 0,601 & 0,360 & $\mathrm{x}$ & 0,000 \\
Micrutalis binaria & 0,785 & 0,705 & 0,630 & 0,241 & 0,658 & $\mathrm{x}$ \\
\hline
\end{tabular}

Agradecimentos. Esse trabalho recebeu apoio do Programa Ecológico de Longa Duração (PELD/Caatinga) e da Coordenação de Aperfeiçoamento de Pessoal de Nível Superior (CAPES). Agradecemos a Alexandre Vasconcellos (UFPB) e aos revisores anônimos pelas contribuições ao manuscrito.

\section{REFERÊNCIAS BIBLIOGRÁFICAS}

Aguiar, C. M. L. 2003. Utilização de recursos florais por abelhas (Hymenoptera, Apoidea) em uma área de Caatinga (Itatim, Bahia, Brasil). Revista Brasileira de Zoologia 20(3):457-467.

Agutar, C. M. L.; Santos, G. M. M.; Martins, C. F. \& Presley, S. J. 2012. Trophic niche breadth and niche overlap in a guild of flower-visiting bees in a Brazilian dry forest. Apidologie, doi:10.1007/s13592-012-0167-4.

Albrecht, M. \& Gotelli, N. J. 2001. Spatial and temporal niche partitioning in grassland ants. Oecologia 126(1):134-141.

AndRAde-Lima, D. 1981. The caatinga dominium. Revista Brasileira de Botânica 4:149-153.

Bartlett, C. R.; Deitz, L. L.; Rothschild, M. J. \& Wallace, M. S. 2008. Treehopper diversity (Hemiptera: Membracidae) of little Orleans, Allegany Co. Maryland. Proceeding of the Entomological Society of Washington 110(1):130-143.

Blüthgen, N. \& KLeIN, A-M. 2011. Functional complementarity and specialisation: the role of biodiversity in plant-pollinator interactions. Basic and Applied Ecology 12(4):282-291.

Capello, S.; Marchese, M. \& Wysiecki, M. L. 2012. Feeding habits and trophic niche overlap of aquatic Orthoptera associated with macrophytes. Zoological Studies 51(1):51-58.
Carvalho, J. C. \& Gomes, P. 2001. Food habits and trophic niche overlap of the red fox, european wild cat and common genet in the Peneda-Gerês National Park. Galemys 13(2):39-48.

Colwell, R. K. \& Futuyma, D. J. 1971. On the measurement of niche breadth and overlap. Ecology 52(4):567-576.

D'Almeida, J. M. \& Almeida, J. R. 1998. Nichos tróficos em dípteros caliptrados, no Rio de Janeiro, RJ. Revista Brasileira de Biologia 58(4):563-570.

D'Avila, M. \& MarchinI, L. C. 2008. Análise faunística de himenópteros visitantes florais em fragmentos de cerradão em Itirapina, SP. Ciência Florestal 18(2):271-279.

Del-Claro, K. 2004. Multitrophic Relationships, Conditional Mutualisms, and the Study of Interaction Biodiversity in Tropical Savannas. Neotropical Entomology 33(6):665-672.

Del-Claro, K. \& Oliveira, P. S. 1993. Ant-Homoptera interaction: do alternative sugar sources distract tending ants? Oikos 68:202-206. 1999. Ant-Homoptera interactions in a neotropical savanna: the Honeydew-producing treehopper, Guayaquila xiphias (Membracidae), and its associated ant fauna on Didymopanax vinosum (Araliacea). Biotropica 31(1):135-144.

2000. Conditional outcomes in a neotropical treehopperant association: temporal and species-specific variation in an protection and homopteran fecundity. Oecologia 124(2): 156-165.

Del-Claro, K.; Byk, J.; Yugue, G.M. \& Morato, M.G. 2006. Conservative benefits in an ant-hemipteran association in the Brazilian tropical savanna. Sociobiology 47(2):415-421.

Dirzo, R.; Young, H. S.; Mooney, H. A. \& Ceballos, G. 2011. Seasonally Dry Tropical Forests: Ecology and Conservation. Washington, Island Press. 394p. 
Fernandes, G. W.; Fagundes, M.; Greco, M. K. B.; Barbeitos, M. S. \& SANTOS, J. C. 2005. Ants and their effects on an insect herbivore community associated with the inflorescences of Byrsonima crssifolia (Linnaeus) H.B.K. (Malpighiaceae). Revista Brasileira de Entomologia 49(2):264-269.

Funkhouser, W. D. 1950. Homoptera. Family Membracidae. Genera Insectorum 208:1-383.

Graciani, C.; Garcia, F. R. M. \& Costa, M. K. M. 2005. Análise faunística de gafanhotos (Orthoptera, Acridoidea) em fragmento florestal próximo ao rio Uruguai, município de Chapecó, Santa Catarina. Biotemas 18(2):87-98.

Gusmão, M. A. B. \& Creão-Duarte, A. J. 2004. Diversidade e análise faunística de Sphingidae (Lepidoptera) em área de brejo e Caatinga no estado da Paraíba, Brasil. Revista Brasileira de Zoologia 21(3):491-498.

HANSKI, I. 1983. Coexistence of competitors in patchy environment. Ecology 64(3):493-500

HERnÁNDEZ, M. I. M. 2007. Besouros escarabeíneos (Coleoptera: Scarabaeidae) da Caatinga Paraibana, Brasil. Oecologia Brasiliensis 11(3): 356-364

HuRlBerT, S. H. 1978. The measurement of niche overlap and some relatives. Ecology 59(1):67-77.

Iannuzzi, L.; Maia, A. C. D.; Nobre, C. E. B.; Suzuki, D. K. \& Muniz, F. J. A. 2003. Padrões locais de diversidade de Coleoptera (Insecta) em vegetação de Caatinga. In: LeAL, I. R.; TABARELLI, M. \& SiLVA, J. M. C. eds. Ecologia e Conservação da Caatinga. Recife, Editora Universitária da UFPE. p.367-390.

Johnson, M. P. \& Freytag, P. H. 1997. Treehoppers (Homoptera: Membracidae) on Pin Oak in Kentucky. Journal of the Kansas Entomological Society 70(1):21-30.

Krebs, C. J. 1999. Ecological Methodology. 2ed. New York, Addison Wesley Educational Publishers. 624p.

Lawton, J. H.; Bignell, D. E.; Bolton, B.; Bloemers, G. F.; Eggleton, P.; Hammond, P. M.; Hodda, M.; Holt, R. D.; Larsen, T. B.; Mawdsley, N. A.; Stork, N. E.; Srivastava, D. S. \& Watt, A. D. 1998. Biodiversity inventories, indicator taxa and effects of habitat modification in tropical forest. Nature 391:72-76.

Leal, I. R; Tabarelli, M. \& Silva, J. M. C. 2003. Ecologia e Conservação da Caatinga. Recife, Ed. Universitária da UFPE. 882p.

Lin, C-P.; Danforth, B. N. \& Wood, T. K. 2004. Molecular phylogenetics and evolution of maternal care in membracine treehoppers. Systematic Biology 53(3):400-421.

Lopes, B. C. 1995. Treehoppers (Homoptera, Membracidae) in Southeastern Brasil: use of host plants. Revista Brasileira de Zoologia 12(3):595-608

MacArthuR, R. H. 1965. Patterns of species diversity. Biological Reviews 40(4):510-533.

MagurRan, A. E. 2011. Medindo a diversidade biológica. Curitiba, Editora UFPR. 261p.

Maia, A. C. D.; Iannuzzi, L.; Nobre, C. E. B. \& Albuquerque, C. M. 2003. Padrões locais de diversidade de Cerambycidae (Insecta, Coleoptera) em vegetação de Caatinga. In: LEAL, I. R.; TABARELLI, M. \& Silva, J. M. C. eds. Ecologia e Conservação da Caatinga. Recife, Editora Universitária da UFPE. p.391-433.

Majumdar, S.; Chaki, K. K. \& MisRa, K. K. 2011. Niche breadth and overlap measures of sarcosaprophagos flies exploiting human settlements. Proceedings of the Zoological Society 64(2):87-95.

Marchiori, C. H.; Caldas, E. R. \& Almeida, K. G. S. 2003. Succession of Scarabaeidae on bovine dung in Itumbiara, Goiás, Brasil. Neotropical Entomology 32(1):173-176.

Mason, C. E. \& Loye, J. E. 1981. Treehoppers (Homoptera: Membracidae) collected at multiple levels in a deciduous woodlot in Delaware. Entomological News 92(2):64-68.

MAY, R. M. 1975. Some notes on estimating the competition matrix alpha. Ecology 56(3):737-741.

Miranda, M. P.; Lopes, J. R. S.; Nascimento, A. S.; Santos, J. L. \& CAVICHIOLI, R. R. 2009. Levantamento populacional de cigarrinhas (Hemiptera: Cicadellidae) associadas à transmissão de Xylella fastidiosa em pomares cítricos do Litoral Norte da Bahia. Neotropical Entomology 38(6):827-833.
Moreira, V. S. \& Del-Claro, K. 2005. The outcomes of an anttreehopper association on Solanum lycocarpum St. Hill: increased membracid fecundity and reduced damage by chewing herbivores. Neotropical Entomology 34(6):881-888.

Paraíba/Governo do estado da Paraíba. 1985. Atlas Geográfico do estado da Paraíba. João Pessoa, Grafset. 100p.

PianKa, E. R. 1974. Niche overlap and diffuse competition. Proceedings of the National Academy of Sciences 71(5):2141-2145.

Prado, D. E. 2003. As Caatingas da América do Sul. In: Leal, I. R.; M. Tabarelli \& J. M. C. Silva eds. Ecologia e Conservação da Caatinga. Recife, Editora Universitária da UFPE. p.3-74.

Prieto, C. \& Dahners, H. W. 2009. Resource utilization and environmental and spatio-temporal overlap of a hilltopping Lycaenid butterfly community in the Colombian Andes. Journal of Insect Science 9(16): 1-12.

Ringenberg, R.; Lopes, J. R. S.; Botton, M.; Azevedo-Filho, W. S. \& Cavichioli, R. R. 2010. Análise Faunística de Cigarrinhas (Hemiptera: Cicadellidae) na Cultura da Videira no Rio Grande do Sul. Neotropical Entomology 39(2):187-193.

Santana, J. A. S. \& Souto, J. S. 2006. Diversidade e estrutura fitossociológica da Caatinga na Estação Ecológica do Seridó-RN. Revista de Biologia e Ciências da Terra 6(2):232-242.

Santos, G. M. M. \& Presley, S. J. 2010. Niche overlap and tempora activity patterns of social wasps (Hymenoptera: Vespidae) in a Brazilian Cashew Orchard. Sociobiology 56(1):121-131.

Santos, J. C.; Almeida-Cortez, J. S. \& Fernandes, G. W. 2011. Richness of gall-inducing insects in the tropical dry forest (caatinga) of Pernambuco. Revista Brasileira de Entomologia 55(1):45-54

Santos, G. M. M.; Carvalho, C. A. L.; Aguiar, C. M. L.; Macêdo, L. S. S. R. \& Mello, M. A. R. 2012. Overlap in trophic and temporal niches in the flower-visiting bee guild (Hymenoptera, Apoidea) of a tropical dry forest. Apidologie, doi:10.1007/s13592-012-0155-8.

Schoereder, J. H. \& Coutinho, L. M. 1991. Atividade forrageira e sobreposição de nichos tróficos em formigas do gênero Atta (Hymenoptera, Formicidae) em Cerrado. Revista Brasileira de Entomologia 35(2):229-236.

Silveira-Neto, S.; Nakano, O.; Barbin, D. \& Villla-Nova, N. A. 1976. Manual de Ecologia dos Insetos. Piracicaba, Editora Agronômica Ceres. 419p.

Tavares, A. F. \& Williams, D. D. 1990. Life history, diet, and niche overlap of three sympatric species of Elmidae (Coleoptera) in a stream. The Canadian Entomologist 122(3):563-577.

Teston, J. A. \& Corseuil, E. 2004. Diversidade de Arctiidae (Lepidoptera, Arctiidae) capturados com armadilha luminosa em seis comunidades no Rio Grande do Sul, Brasil. Revista Brasileira de Entomologia 48(1):77-90.

Teston, J. A. \& Delfina, M. C. 2010. Diversidade de Arctiidae (Lepidoptera, Arctiidae) em área alterada em Altamira, Amazônia Oriental, Pará, Brasil. Acta Amazonica 40(2):387-396.

Thompson, J. N. 2012. O futuro dos estudos plantas-animais. In: Del-Claro, K. \& Torezan-Silingardi, H. M. eds. Ecologia das Interações Plantas-Animais: uma abordagem ecológicoevolutiva. Rio de Janeiro, Technical Books. p.305-333.

Vasconcellos, A.; Andreazze, R.; Almeida, A. M.; Arauo, H. F. P. Oliveira, E. S. \& Oliveira, U. 2010a. Seasonality of insects in a semi-arid Caatinga of northeastern Brazil. Revista Brasileira de Entomologia 54(3):471-476

Vasconcellos, A.; Bandeira, A. G.; Moura, F. M. S.; Araujo, V. F. P. Gusmão, M. A. B. \& Constantino, R. 2010b. Termite assemblages in three habitats under different disturbance regimes in the semi-arid Caatinga of NE Brazil. Journal of Arid Environments 74(2):298-302.

ViAnA, B. F. 1999. A comunidade de abelhas (Hymenoptera: Apoidea) das dunas do Rio São Francisco, Bahia, Brasil. Anais da Sociedade Entomológica do Brasil 28(4):635-645

Wallace, M. S. \& Maloney, S. M. 2010. Treehopper (Hemiptera: Membracidae) biodiversity and seasonal abundance in the Pocono Till Barrens, Long Pond, Pennsylvania. Entomological Society of Washington 112(2):281-294.

Wood, T. K. 1993. Diversity in the New World Membracidae. Annual Review of Entomology 38:409-435. 